\title{
Comunicación y estructura invisible de poder: fondos de inversión en el accionariado de las empresas mediáticas
}

\section{Communication and invisible power structure: Investment funds among the share-holders of media companies}

\author{
Rosalba Mancinas-Chávez; Noelia Ruiz-Alba; Cristina Martín-Jiménez
}

Cómo citar este artículo:

Mancinas-Chávez, Rosalba; Ruiz-Alba, Noelia; Martín-Jiménez, Cristina (2019). “Comunicación y estructura invisible de poder: fondos de inversión en el accionariado de las empresas mediáticas". El profesional de la información, v. 28, n. 6, e280601.

https://doi.org/10.3145/epi.2019.nov.01

Artículo recibido el 10-06-2019 Aceptación definitiva: 22-09-2019

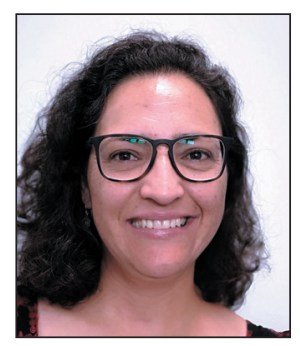

Rosalba Mancinas-Chávez $\square$ http://orcid.org/0000-0002-4218-2338

Universidad de Sevilla, Departamento de Periodismo II

Américo Vespucio, s/n. Isla de la Cartuja, 41092 Sevilla, España

rmancinas@us.es

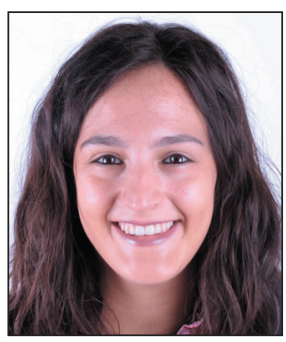

Noelia Ruiz-Alba

https://orcid.org/0000-0001-9438-5519

Universidad de Sevilla, Departamento de Periodismo II

Américo Vespucio, s/n. Isla de la Cartuja, 41092 Sevilla, España noeliaruizalba@gmail.com

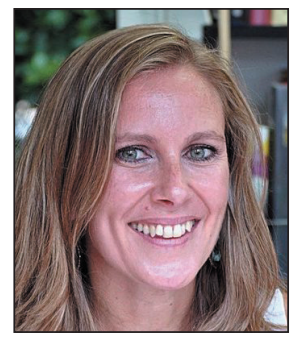

Cristina Martín-Jiménez

https://orcid.org/0000-0001-6518-1808

Universidad de Sevilla, Departamento de

Periodismo II

Américo Vespucio, s/n. Isla de la Cartuja,

41092 Sevilla, España

crismjpress@gmail.com

\section{Resumen}

En la dinámica económica del siglo XXI las empresas más valoradas en bolsa son las cinco tecnológicas Alphabet (Google), Amazon, Facebook, Apple y Microsoft, conjunto conocido como GAFAM. Tienen una fuerte vinculación con los grupos de comunicación, han pasado de ser meras plataformas necesarias para el periodismo digital y todo tipo de contenidos culturales a convertirse en productores comunicativos. En este trabajo se pretende indagar en el accionariado de estas cinco compañías con el fin de demostrar la coincidencia de ciertos fondos de inversión en todas ellas. Probando la maniobra lampedusiana se verá que estos fondos están presentes también en los conglomerados mundiales de la comunicación y aparecen detrás de toda clase de industrias. Esta composición accionarial no es secreta pero se requiere de un esfuerzo de indagación considerable para localizarla, lo que refuerza la idea de la estructura invisible de poder que desarrollaremos en este trabajo.

\section{Palabras clave}

Estructura invisible; Fondos de inversión; Poder; Medios de comunicación; Empresas tecnológicas; Google; Facebook; Amazon; Apple; Microsoft; GAFAM.

\section{Abstract}

Of all the companies which have managed to thrive within the tumultuous economic dynamics of the $21^{\text {st }}$ century, those called GAFAM (Google, Apple, Facebook, Amazon, and Microsoft) are the most valuable group (as per market capitaliza- 
tion). GAFAM is intricately linked to various media outlets as these corporations no longer are platforms for digital journalism (and culture) only: they have evolved into content media producers in their own right. The research presented herein seeks to identify and compare the shareholders of these five companies and demonstrate the repeated presence of the same few investment funds via the execution of the "change-everything-so-that-everything-remains-unchanged" strategy (e.g., 'Lampedusian maneuver' in The Leopard). To know the shareholder composition, while it is not a "secret", requires an exercise of complex research as it is dificult to uncover the invisible power structure shadowing GAFAM.

\section{Keywords}

Invisible structure; Investment funds; Power; Mass media; Technology companies; Google; Facebook; Amazon; Apple; Microsoft; GAFAM.

\section{Introducción}

A partir del concepto de estructura invisible de Bordieu (1996) se plantea esta investigación que aborda el conocimiento del poder y sus estructuras de comunicación en las sociedades democráticas. El concepto no es nuevo, tiene un origen próximo en la Escuela de Frankfurt y se ha estudiado en varias ocasiones a lo largo del siglo XX.

La Historia demuestra que la especie humana

"ha conformado estructuras de poder socioeconómico-mediáticas desde el origen de las civilizaciones" (Reig, 2010).

Es un fenómeno natural en el devenir de la humanidad, en democracia se invisibiliza el poder, la estructura dominante se difumina hasta llegar a ser completamente invisible a la mirada de la sociedad.

En el Senado Romano se observaba un poder visible conformado por los miembros del estamento patricio con alta preeminencia en la escala social, poseedores de tierras y otras propiedades, que acudían como senadores a defender personalmente sus intereses. Pero la realidad histórica — tal y como ya empezó a mostrar Roma por no ir más atrás - enseña que poco a poco ese poder visible le fue cediendo el paso a la política y al político profesional en primera línea mientras que el elemento esencial del poder se desplazaba hasta alcanzar un lugar en la sombra (Grimal, 1990; Gombrich, 1999; Touchard, 2006; Sabine, 1973).

La estructura dominante se difumina hasta llegar a ser completamente invisible a la mirada de la sociedad

Weber (2014) afirma que, desde el punto de vista sociológico, "el poder es amorfo", no sólo invisible. El poder se ha ido invisibilizando conforme ha avanzado la historia de la humanidad y, en concreto, su actividad mercantil.

En el siglo XVIII, por ejemplo, la actividad política y el poder político estaban ya en su cénit, y no era extraño que a los edificios parlamentarios acudieran grupos de poder más o menos en la sombra para "asesorar" a ciertos políticos sobre cómo deberían actuar. Esos grupos son ahora conocidos como lobbies (Castellani, 2018).

En estos momentos del siglo XXI se está planteando un doble fenómeno. Por un lado, la punta del iceberg llamada poder político sigue ahí, a la vista de todo ciudadano consciente. Y también persiste la parte oculta del iceberg, la estructura invisible de poder, más escondida y poco o nada accesible a los ojos del ciudadano. Prueba de ello -en lo que a la estructura comunicacional se refiere- es que, en España, según la Comisión Nacional de los Mercados y la Competencia (CNMC)

"el único punto en el que no hubo consenso para firmar el código ético de las empresas del Ibex-35 fue el de hacer pública su inversión en los medios de comunicación” (Del-Castillo, 2018; González-Pazos, 2019).

Este misterio sobre los propietarios de los medios de comunicación es posible gracias a la connivencia del poder político y económico (Reig, 2015). La implantación paulatina del neoliberalismo ha intensificado el fenómeno de las puertas giratorias (Castellani, 2018) -políticos que pasan a ocupar altos cargos en empresas y viceversa-, al tiempo que se ha producido una llegada explícita de nuevas caras a la política procedentes del mundo "oculto", desde estamentos financieros, por ejemplo.

En el caso de la multinacional Abengoa - denunciada por los medios de comunicación como una de las empresas más politizadas del Ibex 35 (Romera, 2015) - poco antes de estallar la crisis que estuvo a punto de acabar con ella, tenía en su consejo de administración a Ricardo Martínez-Rico, exsecretario de Estado de Presupuestos con el PP entre 2003 y 2004; Josep Borrell, exministro de Obras Públicas con el PSOE, expresidente del Parlamento Europeo y ministro de Asuntos Exteriores, Unión Europea y Cooperación en el Gobierno de Pedro Sánchez; Juan Verde, exasesor de Barack Obama; Luis Solana, exdiputado socialista; y Javier Rupérez, exdiputado, exsenador del PP y exembajador en EUA entre 2000 y 2004. También han trabajado en el grupo José Terceiro, ex subsecretario de la Presidencia del Gobierno de Felipe González, que fue vicepresidente de Abengoa entre 2007 y 2015, y Alberto Aza, exjefe de la Casa Real.

Otro caso vinculado con el salto desde la política a la banca es el de José Manuel Durão Barroso, primer ministro de Portugal entre 2002 y 2004, expresidente de la Comisión Europea (CE), quien al finalizar su cargo en la CE fue nombrado presidente no ejecutivo de Goldman Sachs International en Londres (EFE, 2016). 
No es una cuestión de partidos políticos, es la dinámica de mercado, donde se observa la presencia de políticos de uno y otro signo (Romera, 2015).

El riesgo de las puertas giratorias, según Castellani (2018), es la toma de las decisiones públicas determinada por intereses privados. En materia de comunicación, esta vinculación entre el poder político y económico perjudica finalmente al pluralismo y daña los pilares de la democracia (Reig, 2015).

El diario The guardian (2011) denunciaba que se estaban llevando a cabo pactos entre la política y la banca que beneficiaban a esta última como el Proyecto Merlín ${ }^{1}$.

En España se puede hablar de puertas giratorias en el caso de dos expresidentes españoles, Felipe González (PSOE) y José María Aznar ( $P P)$, que se han introducido en este mundo de la invisibilidad para el ciudadano común. Felipe González ha sido consejero de Gas Natural Fenosa desde 2010 hasta 2015 (Europa Press, 2015). José María Aznar es consejero del conglomerado mundial de comunicación News Corporation desde el año 2006 (Nieto, 2006).

En esta dinámica de ida y vuelta entre el poder político y el económico, la estructura invisible de poder -considerando, sobre todo, a la banca y a los fondos de inversión, tan relacionados con la banca - sitúa a personas de su entorno en puestos de la alta política; personalidades con poca o ninguna trayectoria política terminan presidiendo un país cuando su dedicación vital ha estado, en principio, centrada en las actividades financieras. En esta línea está el ejemplo del presidente de Francia, que procede del sector bancario como director administrativo de Rothschild \& Company (Rubin, 2017).

Situando a personas de su entorno en puestos de la alta política, personalidades con poca o ninguna trayectoria política terminan presidiendo un país cuando su dedicación vital ha estado casi centrada en las actividades financieras

Asimismo, el que fuera primer ministro de Italia en 2011, Mario Monti, no pertenecía a partido alguno, poseía un currículum político escaso, pero había estado ligado a diversas grandes empresas como la banca norteamericana Goldman Sachs, que, a su vez, mantiene una alianza de referencia con el grupo de comunicación argentino Clarín (Reig, 2015).

En este artículo se pretende abordar como elemento crucial de esa estructura invisible de poder los fondos de inversión y su articulación con el mundo mediático. Nos hemos centrado en las cinco empresas tecnológicas (Alphabet-Google, Facebook, Amazon, Microsoft y Apple) porque han relevado en el liderazgo a otras industrias ocupando los primeros lugares en la lista de empresas con mayor cotización en bolsa, como veremos más adelante.

Aunque el análisis de este trabajo se ciñe al sector de la comunicación, esos fondos de inversión tienen como seña de identidad la ubicuidad y, en algunos casos, se ha visto evidenciado el poder que pueden ejercer sobre autoridades políticas. Por ejemplo, en la historia reciente de Argentina se ha experimentado una dependencia de los mismos (Reig, 2015): durante la presidencia de Cristina Fernández de Kirchner (2007-2015) Argentina se endeudó con el fondo Elliott Management Corporation, encabezado por Paul Singer. Este fondo de inversión tiene vínculos accionariales con Interpublic Group, $21^{\text {st }}$ Century Fox, Time-Warner, e Interpublic Group, entre otros.

Asimismo, la presidenta Fernández de Kirchner acometió la medida gubernamental de nacionalizar la empresa YPF, filial de la multinacional del petróleo Repsol. Uno de los accionistas de Repsol es el fondo de inversión BlackRock, omnipresente, como veremos también, en el sector de la comunicación (Reig, 2015). No es extraño que la opinión pública internacional tuviera un posicionamiento en contra de gobiernos como el de Fernández de Kirchner, en su momento, por los mensajes difundidos desde los medios de comunicación participados por inversores con intereses en Repsol.

Para la mayoría de los ciudadanos esta dinámica permanece oculta, por lo que sería necesaria una "alfabetización" sobre ella, puesto que la invisibilidad que analizamos no es ajena a sus vidas. Bordieu (1996) habla de una estructura invisible como una fuerza del mercado, que hace funcionar la competencia movida por unas relaciones de poder ocultas. Esta estructura no es advertida por los espectadores ni por los periodistas. Los periodistas notan sus efectos pero no ven hasta qué punto el peso relativo de la institución en la que trabajan incide sobre ellos

"estructuras invisibles que son un poco, como la fuerza de gravitación, cosas que nadie ve pero que hay que suponer para comprender lo que sucede" (Bordieu, 1996).

\subsection{Poder y comunicación}

El poder precisa de unos mensajes que lo justifiquen, la misión del poder es mantenerse en el poder y la obligación del contrapoder es intentar romper o cambiar la estructura (Reig, 2004).

"Poder es algo más que comunicación, y comunicación es algo más que poder. Pero el poder depende del control de la comunicación, al igual que el contrapoder depende de romper dicho control” (Castells, 2009).

En ese sentido, ¿cuál es el papel de los medios de comunicación en democracia? Mucho se habló del "cuarto poder" en el siglo XIX y se retomó el término en los años sesenta y setenta en Estados Unidos con casos como el Watergate, en los que el periodismo desempeñó esa labor de vigilante del poder político y llegó a derribar al presidente Richard Nixon. Sin 
embargo, Ramonet (2003) advierte que los medios de comunicación no son el cuarto poder, los pone en segundo lugar, después del poder económico. Reig (2004) va un poco más allá y afirma que los medios de comunicación están inmersos en el poder económico, forman parte del mismo a través de conexiones complejas en el accionariado y en los consejos de administración (Reig, 2010).

Según Foucault (1997), el poder no se posee, se ejerce. No es una propiedad, es una estrategia. La comunicación de masas "se conforma y gestiona mediante relaciones de poder enraizadas en el negocio de los medios de comunicación y en la política del estado" (Castells, 2009).

En democracia el poder permanece oculto, los ciudadanos participamos de la elección de los gobernantes, que, según Foucault,

“sabemos perfectamente que no son quienes detentan el poder" (Foucault, 1997, p. 15).

Sabemos quién no tiene el poder, pero desconocemos quién lo tiene. Intuimos que hay una estructura de poder:

"nadie, hablando con propiedad, es su titular y, sin embargo, se ejerce en determinada dirección, con unos a un lado y los otros en el otro; no sabemos quién lo tiene exactamente, pero sabemos quién no lo tiene" (Foucault, 1997).

Distinta es la opinión de Castells (2009), que afirma:

"el poder se basa en el control de la comunicación y la información, ya sea el macropoder del estado y de los grupos de comunicación o el micropoder de todo tipo de organizaciones".

Todos los autores coinciden en la importancia del control de la comunicación por parte del poder. Por ello, cabe preguntarse si los avances en las tecnologías de la información han significado un cambio en el control de la comunicación y si representan la democratización de la información como tantas veces ha llegado a suponerse.

En principio, la aparición de las tecnologías de la información y de la comunicación ha transformado el sistema comunicativo global. Google, Apple, Facebook y Amazon, conocidos con el acrónimo de GAFA', al que se une Microsoft -GA$F A M$ - emergieron en internet ocupando el espacio de las empresas comunicativas tradicionales. Estos cinco gigantes se han convertido en nuevos canales comunicativos, es decir, en las

"pasarelas y plataformas necesarias que dirigen el tráfico hacia determinados contenidos, frente a los grupos de comunicación tradicionales” (Miguel-De-Bustos; Casado-Del-Río, 2016).

Tejedor-Calvo (2010) subraya que

"en un tiempo reducido las redes sociales han pasado de ser un sistema dedicado a la formación de comunidades online a tener un protagonismo destacado dentro de los cibermedios, llegando incluso a asumir funciones de promoción, difusión e información" (2010, p. 617).

Los grandes conglomerados GAFAM, que operan en todos los sectores productivos - desde el papel, las farmacéuticas, las energías o la alimentación hasta la nueva industria aeroespacial一,

"han conseguido superar a los grandes grupos de comunicación tradicionales y convertirse en el agente fundamental del sistema comunicativo cultural mundial” (Miguel-De-Bustos; Casado-Del-Río, 2016).

En un primer momento llegaron a ser la puerta de entrada a casi todos los contenidos culturales comunicativos en la Red, a través de buscadores (Google), redes sociales (Facebook) o grandes almacenes online (Amazon); desde hace unos años han extendido su actividad a la distribución de contenidos comunicativos culturales — plataformas para distribución de contenidos tanto para prensa como para música o audiovisual- y en la actualidad ya desarrollan un papel como creadores de contenidos (Miguel-De-Bustos; Casado-Del-Río, 2016).

Facebook, por ejemplo, ha adquirido tanta relevancia mediática y social que en un informe de 2019 ha reportado un promedio de 1,56 mil millones de usuarios activos diarios. Para esas personas ya no sólo es un lugar donde practicar las relaciones sociales, pues, según el Pew Research Center, el $44 \%$ de los norteamericanos se informa principalmente a través de Facebook (Martín-Jiménez, 2019).

Su potencial fue percibido por Microsoft, que en 2007 compró un 1,6\% de Facebook por 240 millones de dólares y se convirtió en socio de la compañía en otros sectores productivos (Martín-Jiménez, 2019).

En poco más de una década, los GAFAM se han transformado hasta desarrollar funciones propias de la prensa tradicional, como producir y difundir noticias. Son generadores, por tanto, de opinión pública, la cual modifica el pensamiento y la acción de individuos y de comunidades ingentes (Sartori, 2005, p. 176). La función de intermediación que tienen entre los usuarios y los medios de comunicación les confiere un enorme poder en las negociaciones con los productores de contenidos (Miguel-De-Bustos; Casado-Del-Río, 2016).

El análisis y control de los sentimientos, pensamientos y comportamientos de los usuarios de estos nuevos canales y productores comunicacionales, realizados a nivel de big data, permite prever y modificar previamente las reacciones de los receptores, aplicando algunos de los elementos del paradigma de Lasswell (1936). Nos referimos a la pregunta del 
“¿Qué?” - para el control del mensaje- y a la de “¿Con qué efectos?” - formulada para el control de las reacciones de los destinatarios del mensaje-. En 1936, el sociólogo de Chicago ya había descubierto que toda comunicación es intencional porque, como afirmaba en la primera frase del Capítulo 1 de su libro Politics. Who gets what, when, how,

"el estudio de la política es el estudio de la influencia y del influyente".

En este proceso sociológico de control - por parte del Poder - de los mensajes lanzados en los medios de comunicación con el propósito de obtener una respuesta concreta por parte de los receptores, los GAFAM están desarrollando los mismos mecanismos que los medios tradicionales. Que se culpara a Facebook de la victoria electoral de Donald Trump es un ejemplo paradigmático de cómo esta plataforma ha podido condicionar el voto de sus millones de usuarios. Una derrota electoral -la de Hillary Clinton frente a Donald Trump - provocó que Mark Zuckerberg, la cara visible de la compañía, reconociera públicamente que Facebook no es sólo "un distribuidor de noticias" sino "un nuevo tipo de plataforma para el diálogo público". Esta afirmación distó mucho de lo que había defendido sólo unos meses antes, cuando en agosto de 2016 aseguró que la empresa únicamente era una "plataforma tecnológica" y no un medio de información (Martín-Jiménez, 2019). Además, el escándalo de Cambridge Analytics hizo comprender a los usuarios el peligro de compartir todo tipo de información personal en las plataformas digitales que estaban revelando un marcado interés de control y manipulación social.

Los algoritmos de estos gigantes ponen en grave peligro no sólo las democracias sino la libertad personal, pues

"no se trata sólo del control de los individuos sino de la manipulación interesada de sus voluntades, ya sea por motivos comerciales o para que entreguen su voto en democracias pervertidas" (Oliver, 2019).

Como destaca Sartori (2005, p. 176):

"Cuando afirmamos que en las democracias el público se forma una opinión propia de la cosa pública, no afirmamos que el público lo haga todo por sí mismo y solo. Sabemos muy bien, por tanto, que existen 'influyentes' e 'influenciados', que los procesos de opinión van desde los primeros a los segundos, y que en el origen de las opiniones difusas están siempre pequeños núcleos de difusores".

El trabajo de Harold Lasswell y Paul Lazarsfeld en la Mass Communication Research (MCR) estaba dirigido por la elite política-económica de Washington, tanto en su estrategia política como en la mercantil. Sus estudios eran sufragados por la Administración y las grandes corporaciones, que habían descubierto armas sofisticadas y sutiles de manipulación social en los métodos empíricos de la Sociología y de la incipiente ciencia de la Comunicación, y las pusieron a su servicio.

En el elitista núcleo dominante de los GAFAM encontramos hoy a los fondos financieros globales que han surgido de las corporaciones que contrataban y financiaban los servicios de la $M C R$, a principios y mediados del siglo XX. En la actualidad, como entonces, su pretensión es permanecer invisibles y resistentes al análisis de uno de los principales elementos del paradigma de Lasswell: el “¿Quién?".

Por ello, consideramos esencial realizar el análisis de los vínculos entre los GAFAM y los fondos de inversión porque desde la Academia se ha demostrado rigurosa y suficientemente que la naturaleza de la propiedad de los medios de comunicación condiciona el proceso informativo (Schiller, 1976; Curran, 2005; Quirós-Fernández, 2002; Reig, 2011; Mancinas-Chávez, 2008), así como el riesgo para el pluralismo que supone la presencia de accionistas extraños (Reig, 2010) en los medios de comunicación.

\section{Metodología}

Se ha realizado una revisión bibliográfica sobre estructura de la información, estudiando con especial atención las publicaciones de Bourdieu, Foucault, Castells y Reig. En definitiva, la base metodológica sigue los pasos de la Escuela Sevillana de Estructura de la Información (ESEI), fundada por el profesor Ramón Reig.

Además, para obtener información sobre las empresas más poderosas se han consultado en la bolsa de valores Nasdaq las firmas con mayor capitalización bursátil a fecha 3 de junio de 2019. Así, se ha encontrado que Microsoft Corporation, Amazon, Apple, Alphabet y Facebook ocupan las cinco primeras posiciones. Sin embargo, Alphabet Inc. tiene una peculiaridad: cotiza en bolsa con dos clases de acciones y, por ello, ocupa las posiciones cuarta y quinta. Si sumamos los valores de ambos tipos de acciones ocuparía la primera posición.

Posteriormente se han localizado los diez primeros accionistas institucionales de las cinco empresas mencionadas con el fin de ver cuántos coincidían. En total, se han hallado 17 accionistas, constatando que seis de ellos -Vanguard Group, BlackRock, State Street Corporation, FMR, Geode Capital Management, y Northern Trust Corporation- tienen acciones en las cinco empresas GAFAM.

Con esta información y haciendo uso de las aplicaciones Onodo de Fundación Civio y Eurecat, y DataWrapper se han representado a los accionistas en una red y un gráfico de sectores para mostrar de forma sintética su complejo entramado de relaciones.

Se han considerado tres aspectos principalmente en el análisis: el liderazgo económico de los GAFAM en bolsa, la telaraña mediática (Reig, 2010) de las tecnológicas y un análisis de cómo los nuevos medios tienen presencia accionarial de los mismos fondos de inversión que los tradicionales conglomerados mediáticos. 


\section{Resultados}

\subsection{Liderazgo económico de las grandes empresas en bolsa}

Como se ha dicho antes, las cinco empresas con mayor valor bursátil a 3 de junio de 2019 son Microsoft, Amazon, Apple, Alphabet y Facebook. Sobre Alphabet Inc., que cotiza en bolsa con dos clases de acciones, Miguel-De-Bustos y Casado-Del-Río (2016) dicen:

"La escisión de Google en varios grupos, bajo el nombre de Alphabet, es consecuencia de la presión de los inversores que quieren ver y distinguir las diferentes actividades de Google según la rentabilidad presente y futura".

Como dijimos, si se suman los valores de ambos tipos de acciones ocupa la primera posición, tal como se puede observar en la tabla 1.

Tabla 1. Las 20 empresas con mayor capitalización bursátil

\begin{tabular}{|c|c|c|c|c|}
\hline Nombre & $\begin{array}{l}\text { Valor en bolsa (en } \\
\text { miles de millones) }\end{array}$ & País & $\begin{array}{l}\text { Año de entra- } \\
\text { da en bolsa }\end{array}$ & Sector \\
\hline Microsoft Corporation & 979 & EUA & 1986 & Software \\
\hline Amazon.com, Inc. & 863 & EUA & 1997 & Distribución \\
\hline Apple Inc. & 852 & EUA & 1980 & Fabricación de ordenadores \\
\hline Alphabet Inc. & 727 & EUA & $\mathrm{n} / \mathrm{a}$ & Software \\
\hline Alphabet Inc. & 725 & EUA & 2004 & Software \\
\hline Facebook, Inc. & 480 & EUA & 2012 & Software \\
\hline Cisco Systems, Inc. & 235 & EUA & 1990 & Consultoría de equipos de telecomunicaciones \\
\hline Intel Corporation & 201 & EUA & $\mathrm{n} / \mathrm{a}$ & Procesadores \\
\hline Comcast Corporation & 186 & EUA & $\mathrm{n} / \mathrm{a}$ & Servicios de televisión \\
\hline Netflix, Inc. & 156 & EUA & 2002 & Plataforma de vídeo \\
\hline Adobe Inc. & 134 & EUA & 1986 & Software \\
\hline PayPal Holdings, Inc. & 131 & EUA & $\mathrm{n} / \mathrm{a}$ & Servicios de negocios \\
\hline Costco Wholesale Corporation & 110 & EUA & $\mathrm{n} / \mathrm{a}$ & Tienda \\
\hline Broadcom Inc. & 107 & EUA & 2009 & Procesadores \\
\hline Amgen Inc. & 106 & EUA & 1983 & Biotecnología \\
\hline Texas Instruments Incorporated & 103 & EUA & $\mathrm{n} / \mathrm{a}$ & Procesadores \\
\hline Starbucks Corporation & 98 & EUA & 1992 & Restaurantes \\
\hline Nvidia Corporation & 87 & EUA & 1999 & Procesadores \\
\hline Charter Communications & 86 & EUA & 1991 & Servicios de televisión \\
\hline Qualcomm Incorporated & 82 & EUA & 1992 & Equipos de comunicaciones \\
\hline
\end{tabular}

Fuente: Elaborado con datos de Nasdaq (3 de junio de 2019)

Los datos de la tabla 1 permiten constatar el cambio en la dinámica económica, se observa que diez de las 20 empresas con mayor valor en bolsa se dedican a actividades relacionadas con la informática, ya sea porque trabajan en software, procesadores o en la fabricación de ordenadores. El resto ofrece tareas de distribución, consultoría de equipos de telecomunicaciones, servicios de televisión, plataforma de vídeo, negocios, tienda, restauración y biotecnología.

Miguel-De-Bustos y Casado-Del-Río (2016), después del análisis de la emergencia de estas compañías globales en el ámbito de las TIC, afirman que en poco más de una década de existencia, estas empresas ocupan las primeras posiciones del ranking de grupos de comunicación en capitalización bursátil, frente a empresas que llevan dominando décadas el mundo de la comunicación. No sólo se han puesto por encima de los conglomerados mundiales de la comunicación, sino que han superado en valor a otras industrias que ocupaban los primeros lugares del ranking, como Exxon Mobil Corp., General Electric o Wal-Mart Stores (Miguel-De-Bustos; Izquierdo-Castillo, 2019).

\subsection{La telaraña mediática en las tecnológicas}

En el mapa de relaciones del gráfico 1 , se observan los diez primeros accionistas de cada una de las cinco empresas con mayor valor en bolsa. Los círculos rojos de mayor tamaño representan a Vanguard Group, Black-
Diez de las 20 empresas con mayor valor en bolsa se dedican a actividades relacionadas con la informática 


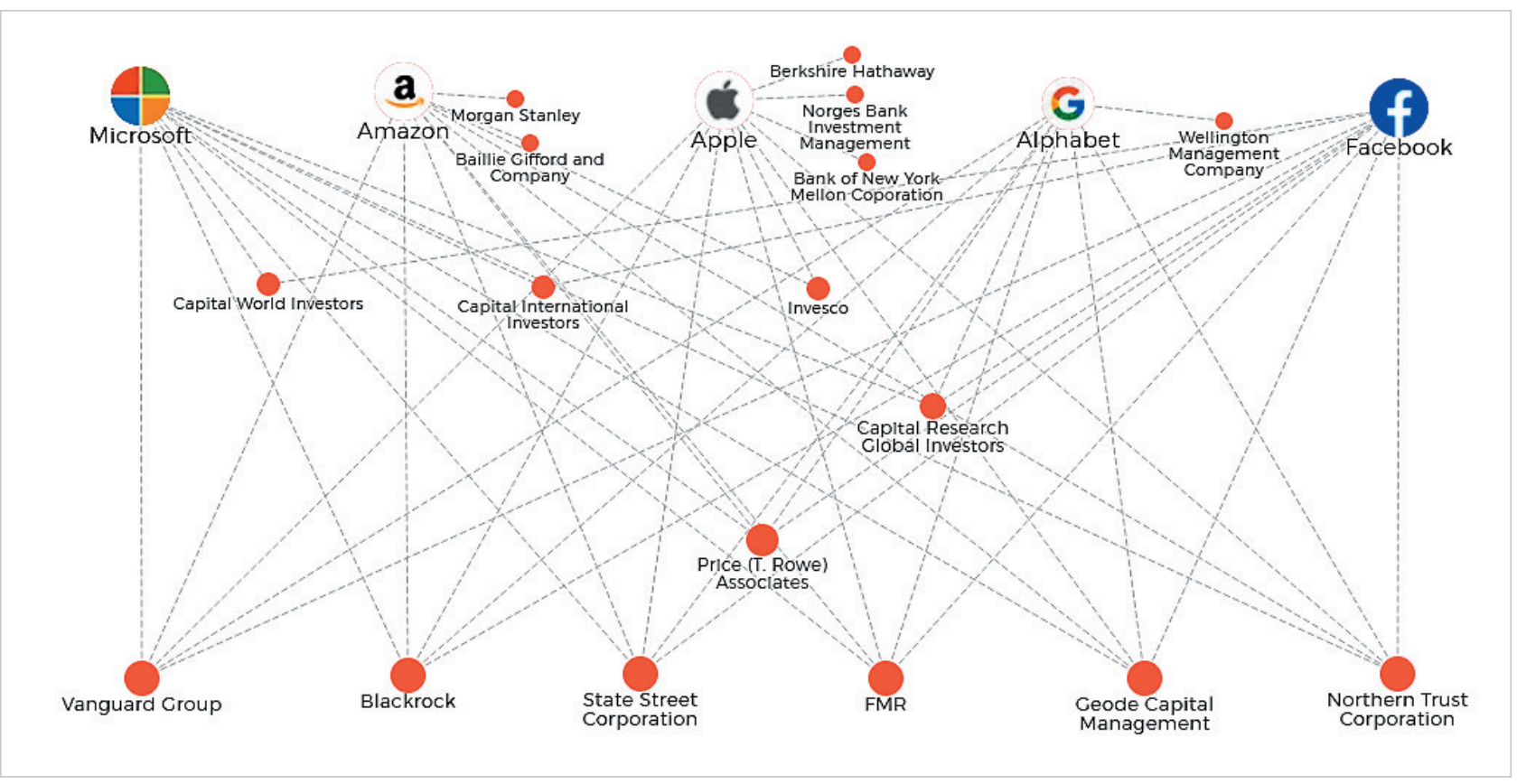

Gráfico 1. Accionistas de los GAFAM

Fuente: Elaborado con datos de Nasdaq y el programa Onodo (Fundación Civio y Eurecat).

Rock, State Street Corporation, FMR, Geode Capital Management y Northern Trust Corporation, los fondos que tienen acciones en las cinco empresas al mismo tiempo. Además, están ordenados según el porcentaje accionarial de cada uno de ellos en los GAFAM, estando ubicados a la izquierda el de mayor cantidad y a la derecha el que menos.

Más arriba se encuentran Price (T. Rowe) Associates y Capital Research Global Investors que poseen acciones en cuatro y tres empresas GAFAM, respectivamente. Las burbujas que se encuentran cerca de los logos de las compañías cuentan con acciones en sólo dos de éstas y las que se ubican a la derecha tienen acciones en la firma a la que acompañan. En los casos en los que hay más de una burbuja junto al logo, como en Amazon, el accionista que tiene mayor porcentaje es el que se encuentra más arriba -Morgan Stanley con el 0,94\%.

Se ha verificado que las cinco compañías objeto de estudio de este trabajo presentan una fuerte presencia de fondos de inversión en su accionariado. Además, como se demuestra en la tabla 2, los principales actores se repiten. Se trata de los seis siguientes: Vanguard Group, BlackRock, State Street Corporation, FMR, Geode Capital Management y Northern Trust Corporation.

Tabla 2. Accionistas que coinciden en los GAFAM

\begin{tabular}{|c|c|c|c|c|c|}
\hline Accionistas & Microsoft & Amazon & Apple & Alphabet & Facebook \\
\hline Vanguard Group & $7,90 \%$ & $6,28 \%$ & $7,36 \%$ & $7,43 \%$ & $7,38 \%$ \\
\hline BlackRock & $6,51 \%$ & $5,21 \%$ & $6,28 \%$ & $0,27 \%$ & $6,14 \%$ \\
\hline State Street Corporation & $4,09 \%$ & $3,36 \%$ & $4,14 \%$ & $3,84 \%$ & $3,85 \%$ \\
\hline$F M R$ & $3,28 \%$ & $3,38 \%$ & $2,20 \%$ & $5,11 \%$ & $4,79 \%$ \\
\hline Geode Capital Management & $1,32 \%$ & $1,98 \%$ & $1,33 \%$ & $1,34 \%$ & $1,29 \%$ \\
\hline Northern Trust Corporation & $1,26 \%$ & $1,00 \%$ & $1,29 \%$ & $1,28 \%$ & $1,17 \%$ \\
\hline
\end{tabular}

Fuente: Elaborado a partir de datos del Nasdaq

En el caso de Vanguard Group, constatamos que es el principal accionista institucional en las cinco empresas, con valores que van desde el 6,28\% en Amazon hasta el 7,9\% en Microsoft. Por su parte, BlackRock también tiene una presencia destacada en las cinco, desde el 5,21\% en Amazon hasta el 6,51\% en Microsoft, distinguiéndose entre el grupo por una menor participación en Alphabet, con un $0,27 \%$. State Street es otro de los fondos con una importante presencia en las cinco, desde el 3,36\% en Amazon hasta el 4,09\% en Microsoft. FMR participa en todas con un 3,28\% en Microsoft, un 3,38\% en Amazon, el 2,2\% en Apple y el 4,79\% en Facebook. De todos los fondos presentes, es el mayor inversor de Alphabet, con un $5,11 \%$.

Geode Capital Management tiene un 1,32\% en Microsoft, un 1,98\% en Amazon, un 1,33\% en Apple, un 1,34\% en Alphabet y un 1,29\% en Facebook. Por último, Geode Capital Management es propietario de un 1,26\% en Microsoft, un $1 \%$ en Amazon, un 1,29\% en Apple, un 1,28\% en Alphabet y un 1,17\% en Facebook. 
Como se observa en la tabla 2, Vanguard Group lidera la cartera de acciones en cada una de las empresas GAFAM, siendo Northern Trust Corporation el último de los fondos en su porcentaje accionarial.

Con respecto al resto de accionistas de estas cinco compañías líderes, encontramos otros grandes grupos financieros que operan, como los precedentes, en todo el globo y que se unen a los seis previamente analizados en la capitalización de los GAFAM. Nos referimos a: Price (T. Rowe) Associates, Capital World Investor, Capital Research Global Investors, Capital International Investors, Invesco, Morgan Stanley, Baillie Gifford and Company, Berkshire Hathaway, Norges Bank Investment Management, Bank of New York Mellon Corporation y Wellington Management Company.

Los nombres de todos los grupos financieros citados aparecen entre los diez primeros accionistas de cada uno de los GAFAM. Hemos comprobado, por lo tanto, que los propietarios principales de las cinco compañías objeto de este estudio solo son 17 fondos. Y, entre estos 17, los seis analizados previamente -Vanguard Group, BlackRock, State Street Corporation, FMR, Geode Capital Management y Northern Trust Corporation- poseen acciones en los cinco GAFAM.

\section{Accionistas de las 5 empresas con más valor en bolsa}

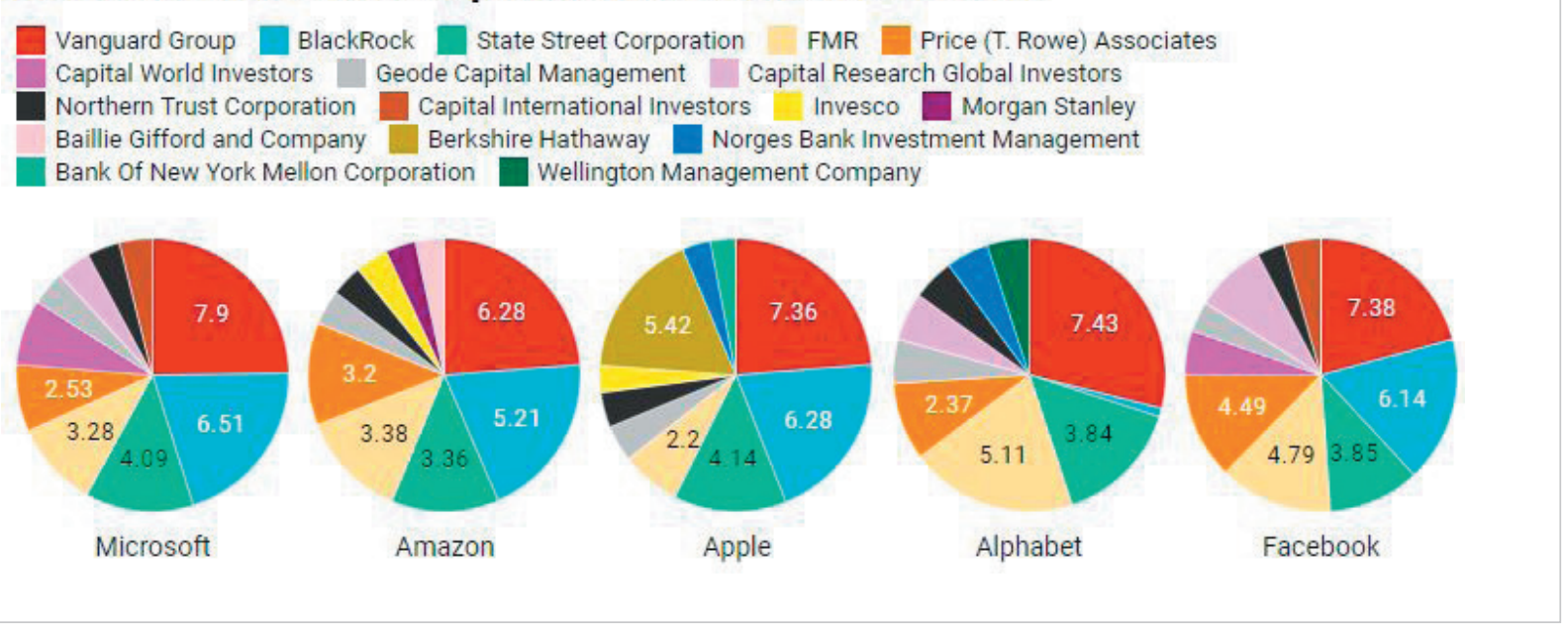

Gráfico 2. Accionistas de los GAFAM

Fuente: Elaborado con datos de Nasdaq y el programa Datawrapper

La telaraña mediática se hace más compleja aún si se intenta investigar la identidad de los dueños de los fondos de inversión que coinciden en los cinco GAFAM. De los seis grupos financieros vinculados por las relaciones de propiedad, solo es posible consultar el accionariado de tres de ellos, porque la otra mitad no ofrece esta información de forma pública. Conforme avanzamos, verificamos la opacidad que los caracteriza, haciéndose aún más presente cuando la investigación pretende profundizar en las identidades, lo que evidencia la pretensión de invisibilidad con la que operan en los mercados y en la res publica. Con los datos disponibles, es posible observar que Vanguard Group, BlackRock y State Street Corporation tienen acciones en BlackRock, State Street Corporation y Geode Capital Management, generando un tejido cada vez más complejo y cerrado, al que solo tienen acceso estos mega fondos de naturaleza endogámica.

Los mismos seis accionistas tienen capital en las cinco empresas GAFAM

Tabla 3. Accionistas de los principales accionistas de los GAFAM

\begin{tabular}{|l|c|c|c|}
\hline \multicolumn{1}{|c|}{ Accionistas } & BlackRock Inc. & State Street Corporation & Geode Capital Management \\
\hline Vanguard Group, Inc. (The) & $5,80 \%$ & $7,66 \%$ & $7,26 \%$ \\
\hline BlackRock Inc. & $4,89 \%$ & $7,52 \%$ & $6,37 \%$ \\
\hline State Street Corporation & $3,33 \%$ & $4,86 \%$ & $4,36 \%$ \\
\hline
\end{tabular}

Fuente: Elaborado a partir de datos del Nasdaq. Obsérvese cómo BlackRock Inc. y State Street Corporation poseen menos del 5\% de su propia compañía.

\subsection{Nuevos medios, mismos dueños}

La economía actual muestra claramente la maniobra lampedusiana (Tomasi-Di-Lampedusa, 2004), "cambiar todo para que todo siga igual", que en términos de periodismo ha sido estudiada con detenimiento por Reig (2004; 2010; 2011; 2015). Los fondos de inversión participan de las principales empresas en bolsa, independientemente de que sean conglomerados clásicos o nuevos medios. 
En este sentido, una característica común entre los grupos de comunicación y los GAFAM es que los mismos fondos de inversión están presentes en buena parte de la propiedad a través de la figura de financiarización (Miguel-De-Bustos; Casado-Del-Río, 2016).

Los conglomerados mediáticos tradicionales como News Corporation, Comcast Corporation, Viacom Inc. o CBS Corporation (Reig, 2011) comparten accionistas mayoritarios con las cinco empresas con mayor valor en bolsa, los GAFAM. No disponemos de datos de The Walt Disney, que al cotizar en la bolsa de Nueva York no tiene accesible esta información.

De este modo, hemos comprobado que Vanguard Group, BlackRock y State Street Corporation coinciden tanto en el accionariado de las nuevas empresas con mayor valor en bolsa como en los tradicionales conglomerados mediáticos.

Tabla 4. Accionistas de los conglomerados mediáticos tradicionales

\begin{tabular}{|l|c|c|c|c|}
\hline Accionistas & News Corp. & Comcast Corp. & Viacom Inc. & $\begin{array}{c}\text { The Walt Disney } \\
\text { Company }\end{array}$ \\
\hline Vanguard Group & $13,45 \%$ & $8,50 \%$ & $11,25 \%$ & $7,07 \%$ \\
\hline BlackRock & $6,59 \%$ & $7,01 \%$ & $7,86 \%$ & $6,83 \%$ \\
\hline State Street Corporation & $4,55 \%$ & $3,80 \%$ & $5,04 \%$ & $4,23 \%$ \\
\hline
\end{tabular}

Fuente: Elaborado a partir de datos del Nasdaq

Al mismo tiempo, en la fuente consultada - Nasdaq- los citados fondos aparecen como accionistas en empresas globales de muy diversos sectores económicos y productivos, por ejemplo, Exxon Mobile (petrolera), Coca-Cola (restauración), McDonald's (restauración), Boeing (aviación), General Motors (automóviles), General Electric (electrodomésticos y armas, entre otros), Helmerich \& Payne (petrolera), Hewlett Packard Enterprise (tecnologías de la información).

Es de esta forma cómo corroboramos que en los sectores en los que operan estos fondos se produce el fenómeno de la "telaraña mediática" (Reig, 2010). Queda demostrado que estos grupos financieros son propietarios de los GAFAM y de medios de comunicación tradicionales y, al mismo tiempo, están presentes en el accionariado de todo tipo de industrias y productos ajenos al ámbito de la comunicación y la información. Su presencia en los grandes conglomerados mediáticos condiciona el contenido periodístico y, por lo tanto, la pluralidad informativa, ya que genera la difusión de mensajes homogéneos y unidireccionales. Como éstos carecen de la perspectiva crítica, consiguen alterar la calidad democrática de las sociedades en las que operan ${ }^{3}$ desde su posición de invisibilidad.

\section{Conclusiones}

La economía del siglo XXI ha dado un giro con relación a la del siglo XX. Las grandes industrias que lideraban el ranking de mayor valor en bolsa han sido sustituidas por conglomerados dedicados a la comunicación. Se trata de cinco compañías cuyo principal negocio son las tecnologías de la información y la comunicación: Google, Amazon, Facebook, Apple y Microsoft (GAFAM). En menos de una década, han pasado de ser meras plataformas digitales a productores de contenidos mediáticos y de las industrias culturales.

La cara visible de estas cinco compañías son empresarios jóvenes, que plantean una nueva forma de comprender e interactuar en las sociedades democráticas. Sin embargo, el estudio detallado de la composición accionarial nos lleva a comprobar la maniobra lampedusiana, por la que los principales accionistas son los mismos fondos de inversión que sostenían a los conglomerados clásicos y los mismos que estaban presentes en las más poderosas industrias del siglo XX, como son las petroleras y las energéticas. Han ido cambiando los nombres, incluso la tipología de sus empresas conforme crecían y ampliaban su campo de operación en el contexto de la globalización, pero la base económica y su mecánica de actuación sigue siendo la misma.

Es decir, son mega compañías que están en el accionariado de todo tipo de sectores productivos y económicos, al mismo tiempo que en el sector de la comunicación y la información. Se mueven en la opacidad y la invisibilidad, presentan una naturaleza de carácter endogámica y su número es muy reducido, como hemos comprobado en nuestro análisis (Mancinas-Chávez; Nogales-Bocio; Yaguache-Quichimbo, 2019).

A lo largo de este trabajo se ha demostrado que los diez primeros accionistas de las cinco empresas más poderosas son 17 fondos de inversión que, a su vez, intercambian accionariado entre ellos. Es una telaraña compleja de intereses económicos y financieros que nos lleva a preguntarnos la viabilidad de la democracia en el siglo XXI. Volvemos a la estructura invisible y al postulado de Foucault: sabemos quién no tiene el poder, pero no sabemos quién lo tiene. Se ha diluido en múltiples polos de poder que conducen a las mismas estructuras. Los hombres -y mujeres- detrás de estos fondos de inversión no se presentan a elecciones, por lo que su participación en el juego democrático no es percibible por la ciudadanía. Están situados por encima de la dinámica democrática, en el plano de la invisibilidad, por lo que su existencia, así como las posibles presiones y condicionamientos a las sociedades en las que desarrollan sus actividades pasan inadvertidas a las audiencias de sus medios, los ciudadanos y los votantes. 


\section{Notas}

1. Se le llamó así a un convenio entre el Gobierno Británico y la banca, supuestamente destinado a financiar empresas al objeto de crear puestos de trabajo. El diario sostenía que se trataba de un acuerdo elaborado por los bancos a su medida, a través de John Varley, ex alto cargo de la banca Barclays, estimulado por el entonces primer ministro James Cameron y por el ministro de Hacienda, George Osborne.

2. Miguel-De-Bustos y Casado-Del-Río (2016) consideran atribuible el término GAFA a Simon Andrews, creador de la agencia londinense de marketing Addictive, por ser el primero en usarlo en 2011. En este artículo hablamos de GAFAM porque incluimos Microsoft.

3. La Escuela Sevillana de Estructura de la Información ha constatado diversos casos en los que se evidencia esta "telaraña".

\section{Referencias}

Bordieu, Pierre (1996). Sobre la televisión. Barcelona: Anagrama. ISBN: 8433905473

Castellani, Ana (2018). "Lobbies y puertas giratorias". Nueva sociedad, democracia y poder en América Latina (NUSO), n. 276.

https://nuso.org/articulo/lobbies-y-puertas-giratorias

Castells, Manuel (2009). Comunicación y poder. Madrid: Alianza editorial. ISBN: 9788420684994

Curran, James (2005). Medios de comunicación y poder. Barcelona: Editorial Hacer. ISBN: 9788488711748

Del-Castillo, Carlos (2018). “¿Quién controla los medios? No quieren que lo sepas, pero hay formas de rastrearlo”. Público, 30 enero.

https://www.publico.es/politica/controla-medios-no-quieren-sepas-hay-formas-rastrearlo.html

EFE (2016). “Goldman Sachs International designa a Durão Barroso presidente no ejecutivo”. EFE, 8 julio.

https://www.efe.com/efe/espana/efeempresas/goldman-sachs-international-designa-a-durao-barroso-presidente-noejecutivo/50000908-2980384\#

Europa Press (2015). “Felipe González deja el consejo de Gas Natural Fenosa”. Europa Press, 27 marzo. https://www.europapress.es/economia/noticia-felipe-gonzalez-deja-consejo-gas-natural-fenosa-20150327154937.html

Foucault, Michel (1997). Un diálogo sobre el poder. Madrid: Alianza editorial. ISBN: 8420618160

Gombrich, Ernst H. (1999). Breve historia del mundo. Barcelona: Península. ISBN: 9788499423470

González-Pazos, Jesús (2019). Medios de comunicación. ¿Al servicio de quién? Barcelona: Icaria. ISBN: 9788498888997

Grimal, Pierre (1990). El helenismo y el auge de Roma. Madrid: Siglo XXI. ISBN: 9788432300660

Lawswell, Harold (1936). Politics who gets what, when and how. New York, London: Whittlesey House. ISBN: 9781 258139599

Mancinas-Chávez, Rosalba (2008). El poder mediático en México. Relaciones entre economía, política y medios de comunicación. Grupo de Investigación en Estructura, Historia y Contenidos de la Comunicación (Grehcco). ISBN: 97884 93059699

Mancinas-Chávez, Rosalba; Nogales-Bocio, Antonia-Isabel; Yaguache-Quichimbo, Jenny-Jovita (2019). “Estructuras de propiedad. Los dueños de la información digital”. En: Romero-Rodríguez, Luis-Miguel; Rivera-Rogel, Diana. La comunicación en el escenario digital. Actualidad, retos y prospectivas. Perú: Pearson, pp. 219-323. ISBN: 9786073248594

Martín-Jiménez, Cristina (2019). "Propietarios de Facebook Inc.: Principales datos estructurales y financieros". Ámbitos. Revista internacional de comunicación, n. 44.

https://doi.org/10.12795/Ambitos.2019.i44.12

Miguel-De-Bustos, Juan-Carlos; Casado-Del-Río, Miguel-Ángel (2016). “Emergencia de los GAFA y cambios en el sistema comunicativo global”. Telos. Revista de pensamiento sobre comunicación, tecnología y sociedad, n. 104, pp. 1-11.

https://telos.fundaciontelefonica.com/archivo/numero104/emergencia-de-los-gafa-y-cambios-en-el-sistemacomunicativo-global

Miguel-De-Bustos, Juan-Carlos; Izquierdo-Castillo, Jessica (2019). “¿Quién controlará la Comunicación? El impacto de los GAFAM sobre las industrias mediáticas en el entorno de la economía digital”. Revista latina de comunicación social, n. 74.

https://doi.org/10.4185/RLCS-2019-1358

Nieto, Ana B. (2006). "Aznar nombrado por Murdoch consejero de News Corporation". Cinco días, 22 junio. https://cincodias.elpais.com/cincodias/2006/06/22/empresas/1150983594_850215.html 
Olier, Eduardo (2019). “Una técnica matemática que dividirá al mundo. Algoritmo, el poder del control social”. Cuaderno Geotecnología, Telos, n. 110, pp. 60-67.

https://telos.fundaciontelefonica.com/wp-content/uploads/2019/04/telos-110-cuaderno-geotecnologia-eduardo-olier.pdf

Quirós-Fernández, Fernando (2002). “La prensa del siglo XXI: el final del perro guardián”. Telos, n. 51.

https://www.academia.edu/7672334/La_prensa_del_siglo_XXI_el_final_del_perro_guardi\%C3\%A1n

Ramonet, Ignacio (2003). La tiranía de la comunicación. Madrid: Temas de debate. ISBN: 9788483065419

Reig, Ramón (2004). Dioses y diablos mediáticos. Cómo manipula el poder a través de los medios de comunicación. Barcelona: Urano. ISBN: 9788479535520

Reig, Ramón (2010). La telaraña mediática, cómo conocerla, cómo comprenderla. Zamora: Comunicación Social. ISBN: 9788492860548

Reig, Ramón (2011). Los dueños del periodismo. Claves de la estructura mediática mundial y de España. Barcelona: Gedisa. ISBN: 9788497846189

Reig, Ramón (2015). Crisis del sistema, crisis del periodismo. Contexto estructural y deseos de cambio. Barcelona: Gedisa. ISBN: 9788497849111

Romera, Javier (2015). “Abengoa tiene cinco políticos en nómina: una de las empresas más politizadas del Ibex 35”. El economista, 26 noviembre.

https://www.eleconomista.es/empresas-finanzas/noticias/7175801/11/15/Abengoa-tiene-cinco-politicos-en-nominauna-de-las-empresas-mas-politizadas-del-lbex.html

Rubin, Alissa J. (2017). “¿Quién es el candidato francés Emmanuel Macron?”. The New York Times, 21 abril. https://www.nytimes.com/es/2017/04/21/quien-es-el-candidato-frances-emmanuel-macron

Sabine, George H. (1973). Historia de la teoría política. México DF: FCE. ISBN: 9789681641993

Sartori, Giovanni (2005). Elementos de teoría política. Madrid: Alianza Editorial. ISBN: 9788420647876

Schiller, Herbert I. (1976). Communications and cultural domination. White Plains. New York: International Art and Science Press. ISBN: 0873320794

Tejedor-Calvo, Santiago (2010). "Web 2.0 en los ciberdiarios de América Latina, España y Portugal". El profesional de la información, v. 19, n. 6.

https://doi.org/10.3145/epi.2010.nov.07

The guardian (2011): "Politicians and bankers: Struggle for power". The guardian, 11 Febr.

https://www.theguardian.com/commentisfree/2011/feb/10/politicians-bankers-struggle-for-power

Tomasi-Di-Lampedusa, Giuseppe (2004). El gatopardo. Madrid: Alianza Editorial. ISBN: 9788420657158

Touchard, Jean (2006). Historia de las ideas políticas. Barcelona: Tecnos. ISBN: 9788430943555

Weber, Max (2014). Economía y sociedad. México DF: FCE. ISBN: 9786071620897

\section{El profesional de la información \\ Servicio de traducciones al inglés}

http://WWW.elprofesionaldelainformacion.com/documentos/traducciones.pdf

Información: Isabel Olea

epi.ioleadgmail.com 